American Journal of Pharmaceutical Education 2020; 84 (5) Article 7693.

\title{
RESEARCH
}

\section{Using a Mixed Methods Approach to Explore Perceptions of Early Learners in Classroom-Based Interprofessional Education Experiences}

\author{
Abigale T. Matulewicz, PharmD, Sharon K. Lanning, DDS, Kelly Lockeman, PhD, \\ Laura M. Frankart, PharmD, MEd, Emily P. Peron, PharmD, MS, Kacie Powers, PharmD, \\ Patricia W. Slattum, PharmD, PhD, Alan W. Dow, MD, MSHA \\ Virginia Commonwealth University School of Pharmacy, Richmond, Virginia \\ Submitted May 23, 2019; accepted September 26, 2019; published May 2020.
}

Objective. To explore attitudes and learning outcomes among early-level health professions students who completed foundational interprofessional education (IPE) courses.

Methods. This study used a mixed methods approach to examine assessment and evaluation data from two student cohorts enrolled in two one-credit, semester-long interprofessional courses taught in fall and spring 2017. Attitudinal changes following the fall course were measured and compared in a retrospective pretest-posttest manner across student disciplines using the Student Perceptions of Interprofessional Clinical Education-Revised Instrument, version 2 (SPICE-R2). Course evaluation comments and narrative reflection assignments for both courses were analyzed qualitatively via data reduction and compilation to identify evidence of learning.

Results. Significant increases in positive student perceptions regarding IPE were found, with variation in the increase seen between professions following the first course. Core themes identified in the narrative reflections demonstrated student learning in interprofessional attitudes, communication, professional identity, collaborative behaviors, and systems of care.

Conclusion. Student attitudes toward interprofessional learning were more positive following completion of a foundational IPE course. In addition, learning in the course shaped students' professional identities, collaborative behaviors, and understanding of systems of care. These findings suggest value in early IPE and directions for better structuring curriculum and timing of IPE.

Keywords: interprofessional education, early learners, attitudes, communication

\section{INTRODUCTION}

Interprofessional education (IPE) is the educational strategy of having two or more professions learn about, from, and with one another, with an end goal of enabling effective collaboration and improving health outcomes. ${ }^{1}$ Although IPE has been integrated into accreditation requirements for the majority of health professions programs, ${ }^{2}$ the best approach to IPE remains uncertain. One source of uncertainty across health professions degree programs is when to introduce learners to IPE. ${ }^{3,4}$

The Accreditation Council for Pharmacy Education (ACPE) Standards 2016 emphasize a requirement of all schools of pharmacy to provide opportunities for IPE, with an ultimate goal of graduates successfully providing patient-centered care in team-oriented settings. ${ }^{5}$ This requirement goes beyond the typical experiences

Corresponding Author: Abigale T. Matulewicz, 410 N 12th St., PO Box 980533, Richmond, VA 23298-0533. Tel: 804-6283867. Fax: 804-828-0343. Email: atmatulewicz@vcu.edu. afforded in experiential learning to emphasize the need for didactic activities focused on exposing students to prescribers and other healthcare professionals in a variety of practice settings. This interprofessional focus should be introduced to early learners, then reinforced throughout the Doctor of Pharmacy (PharmD) program, and ultimately lead to achievement of competency in core interprofessional competencies during advanced pharmacy practice experiences (APPE). While the standard is not explicit in the structure or types of IPE required of schools, emphasis is placed on a balance between simulation and real-world activities with communication, conflict resolution, and professional identity as core concepts.

Experiences described within pharmacy education indicate that a variety of methods have been used to expose students to IPE. Simulation-based experiences, using either low- or high-fidelity equipment, can provide students with a realistic context for interprofessional development, but often are provided as a single, stand-alone 


\section{American Journal of Pharmaceutical Education 2020; 84 (5) Article 7693.}

activity and may require significant technology and resources. ${ }^{6-8}$ Elective courses and workshops with small cohorts of students, typically focused on one facet of interprofessionalism or healthcare, such as medication safety, medication errors, or situation-background-assessment-recommendation (SBAR) training, are also common. ${ }^{9-12}$ Few have enrolled large numbers of students from multiple health professions degree programs using a team-based approach with a traditional lecturebased format. $^{13,14}$

When examining other health professions and their use of IPE, some authors argue that IPE should only be incorporated during the clinical stage of training. ${ }^{15}$ They state that initially exposing learners to interprofessional concepts in the clinical environment provides immediate context to support learning that helps integrate collaborative approaches into future clinical work. This approach also avoids the logistical challenges of implementing IPE in the preclinical setting, though implementation in the clinical stage of training is not without challenges of its own. ${ }^{16,17}$ On the contrary, other authors have suggested that a threaded, longitudinal approach to IPE is most effective. ${ }^{18}$ Creating a longitudinal, threaded approach with activities for preclinical learners can provide foundational knowledge about interprofessional practice prior to entering the clinical environment. ${ }^{19}$ In addition, pre-clinical IPE experiences appear to promote positive attitudes about interprofessional practice and facilitate professional identity development and interprofessional socialization. $^{20-22}$ Many current practitioners did not participate in IPE during their training and thus did not have the opportunity to develop the socialization and teamwork skills common to interprofessional practice until later in their careers. ${ }^{23}$ Earlier IPE exposure may be beneficial for helping health professions students think about their future practice beyond the traditional siloed scope.

At Virginia Commonwealth University (VCU), we sought to thread IPE longitudinally throughout the curricula of health professions degree programs by including foundational, for-credit coursework for preclinical learners in addition to later clinical experiences. Our previous work showed that classroom-based IPE experiences for preclinical learners support interprofessional socialization based on quantitative data, but we have also found that students who lack clinical experience often struggle to see the relationship between interprofessional concepts and the delivery of health care services, which may be a barrier to their learning. ${ }^{22}$ The purpose of this study was to explore attitudes and learning outcomes among early-level health professions students who completed two foundational IPE courses.

\section{METHODS}

This study used a mixed methods approach to examine assessment and evaluation data from two onecredit, semester-long interprofessional courses taught in 2017. Each course enrolled approximately 500 health professions students who were divided into 88 interprofessional teams of about six members each. These teams worked together on activities throughout the semester. Attitudinal change following completion of the first interprofessional course was measured and compared across student disciplines. Narrative reflections and course evaluation comments from both interprofessional courses were then analyzed qualitatively to describe any observed quantitative changes and enhance understanding of the impact of the two courses. This study was approved as exempt by the VCU Institutional Review Board.

The first course, Foundations of Interprofessional Practice, introduced concepts such as roles and responsibilities, interprofessional communication, and teams and teamwork from the core competencies for interprofessional collaborative practice. ${ }^{24}$ In fall 2017 , enrollment included students from seven health professions programs: Bachelor of Science program in dental hygiene, $n=15$; Doctor of Dental Surgery, $n=96$; Master of Health Administration, $\mathrm{n}=17$; Bachelor of Science in nursing; $n=155$; Doctor of Occupational Therapy, $n=42$; Doctor of Pharmacy, $\mathrm{n}=128$; and Doctor of Physical Therapy, $n=53$. At the time of the study, all students were in the first semester of their respective programs except for the nursing and dental hygiene students, who were in their fifth semester.

The second of the two courses, Interprofessional Quality Improvement and Patient Safety, occurred later in students' training and introduced the concepts of quality and safety through the lens of interdependent healthcare collaboration across professions. In spring 2017, enrollment in Interprofessional Quality Improvement and Patient Safety included students from three health professions programs: first-year medical (MD) students, $\mathrm{n}=215$; third-year nursing students, $\mathrm{n}=145$; and secondyear PharmD students $(n=130)$. While the nursing and pharmacy students had completed Foundations of Interprofessional Practice in fall 2015 (more than two years earlier), the medical students had not.

Quantitative data, which had been collected anonymously using Qualtrics (Provo, UT), were obtained from the course evaluation survey for Foundations of Interprofessional Practice. A link to the survey was emailed to students at the end of the course, and participation was strongly encouraged but not required. Besides traditional course evaluation questions, the survey contained an 


\section{American Journal of Pharmaceutical Education 2020; 84 (5) Article 7693.}

attitudinal measure that was administered using a retrospective pretest-posttest design.

The Student Perceptions of Interprofessional Clinical Education-Revised Instrument, version 2 (SPICE-R2) was also administered to students in the Foundations course. The SPICE-R2 is a self-report measure that consists of 10 items rated on a five-point Likert scale. The instrument gauges student attitudes in three domains related to interprofessional collaboration: interprofessional teamwork and team-based practice, roles/responsibilities for collaborative practice, and patient outcomes from collaborative practice. $^{25}$ The SPICE-R2 was selected because of its concise nature, strong psychometric properties, and alignment with the objectives of the course. The original Student Perceptions of Physician-Pharmacist Interprofessional Clinical Education (SPICE) instrument was revised and validated for use in all health professions as SPICE-R. The revised version (ie, SPICER2) addressed observed deficiencies in the original model structure. $^{25-27}$ The SPICE-R2 survey was not administered to students in the Interprofessional Quality Improvement and Patient Safety course for this study because the nursing and pharmacy students had already completed that instrument in the Foundations course in 2015 (data unpublished).

For both the retrospective pretest and posttest, item responses were summed for a total score, and responses for items in each domain were summed to obtain subscale scores. The difference in scores was calculated and classified as the measure of attitudinal change for each respondent. Scores from each time point were compared using a paired samples $t$ test to determine whether there was a significant difference between pretest and posttest scores overall and for each domain. Changes in scores were also compared across professional disciplines using one-way analysis of variance (ANOVA) to determine whether the degree of change was different between students in the various health professions degree programs. Statistical analysis was completed using SAS, 9.4 (SAS Institute, Cary, NC).

Qualitative data came from six sources: four teambased reflection assignments and two course evaluation surveys. In each course, all students completed narrative reflection assignments in their assigned team as part of course activities. Teams were randomized and typically included six to seven students each, with the goal of at least one representative from the larger health professions programs present in Foundations of Interprofessional Practice and a mix of professions present for Interprofessional Quality Improvement and Patient Safety. The Foundations course required students to complete three unique reflection assignments, averaging 200 words each, which were included in the dataset. The Interprofessional Quality Improvement and Patient Safety required students to complete one reflection assignment, which averaged 500 words, and was included in the data set. Guided questions were used to prompt reflections and typically required students to identify two of the most important takeaway points from the course materials or activities completed that would affect their training or healthcare practice. Reflections were graded by course faculty members and were included as part of the course grading structure. To sample these data, blocks of 10 team reflections were randomly selected for qualitative coding and analysis from each of the four sources, creating an initial sample of 40 narrative reflections. Another set of 10 reflections from each source was selected using the same technique to provide a second sample, which was used to ensure saturation.

In addition, an online course evaluation survey was administered in each course at the end of the semester. Each survey instrument included open-ended response items to collect comments and suggestions from individual students about the learning experience. On both course evaluation surveys, students were prompted to answer the question, "What other comments or suggestions do you have about the course?" All of the narrative comments from both course evaluations were included in the analysis, a data set of more than 30,000 words.

For each data source (ie, reflective assignment or course evaluation), an interprofessional dyad from the research team $(n=8)$ was assigned to complete thematic analysis. These two-person teams had all been involved as course directors or faculty members in at least one of the courses. Coding and thematic analysis was done using $\mathrm{G}$ Suite (Google, Mountain View, CA). Each member of each dyad reviewed the first sample from their assigned data source and identified themes. Through a process of data reduction and data compilation, the dyads generated meaningful patterns and codes. ${ }^{28}$ Data were then categorized into themes using an inductive approach, with consensus and saturation achieved by reviewer dyads. ${ }^{29}$

The entire research team then met to discuss the findings of each dyad and come to a consensus regarding overarching themes as a structure to guide reexamination and coding of the data. After agreement on codes among the research team members, individual investigators then recoded the first and second samples from the data sources they had been assigned. The dyad members next met with each other to reach consensus on coding of their data source. Narrative text and the codes assigned to that data were then transferred to one shared spreadsheet for further organization and analyses of the themes across data sources. 


\section{American Journal of Pharmaceutical Education 2020; 84 (5) Article 7693.}

For each of the resulting themes, an individual researcher was selected to organize the coded data into subthemes, define and describe subthemes, and select exemplar quotes. The entire research team then came together to review and confirm the summaries.

\section{RESULTS}

Of the 506 students enrolled in Foundations of Interprofessional Practice in the fall semester, 268 (53\%) submitted a course evaluation. Of these, 243 provided complete responses that could be used in quantitative analysis of the retrospective pretest and posttest SPICER2 measure, but the response rate for this measure varied by student program. Of the 490 students enrolled in Interprofessional Quality Improvement and Patient Safety in the spring, 343 (70\%) completed the course survey. A total of 345 students (160 from the Foundations course and 185 from the Quality Improvement and Patient Safety course) provided open-ended comments about the courses on the evaluation surveys. In each course, all students completed the respective reflection assignments as part of an assigned team.

Overall, students had significantly more positive perceptions about IPE after completing the Foundations of Interprofessional Practice course $(M=40.5, \mathrm{SD}=8.6)$ than before the course $(\mathrm{M}=36.6, \mathrm{SD}=8.1 ; \mathrm{t}(242)=-10.12$, $p<.001$, Cohen $d=.472$ ). Complete descriptive statistics are presented in Table 1. Among the three subscales, the change in student attitudes toward interprofessional roles and responsibilities was greatest $(p<.001$, Cohen $d=1.021)$. Attitudes about teamwork $(p<.001$, Cohen $d=.232$ ) and impact of interprofessional practice on patient outcomes $(p<.001$, Cohen $d=.345)$ both showed low-to-moderate change. The one-way ANOVA revealed a significant main effect for student profession in the degree to which overall perceptions about interprofessional learning changed following completion of the
Foundations course $(\mathrm{F}[6,236]=3.05, p=.007)$. Post hoc tests revealed significant differences between pharmacy and dentistry students and pharmacy and occupational therapy students (Figure 1). Significant main effects for student profession were also observed in the subscales measuring roles and responsibilities for collaborative practice (three items) $(\mathrm{F}[6,236]=2.67, p=.016)$ and patient outcomes from collaborative practice (three items) $(\mathrm{F}[6,236]=3.72, p=.002)$. For roles and responsibilities, post hoc tests indicated that the primary difference was between pharmacy and dentistry students, while variance in attitudes about patient outcomes were observed between pharmacy students and students from dentistry, nursing, and occupational therapy.

\section{Qualitative Analysis}

Five overarching but interrelated themes representing student learning emerged from the qualitative data: interprofessional attitudes, communication, collaborative behaviors, professional identity, and systems of care. These themes, as well as their subthemes, are summarized in Table 2, with supporting exemplar quotes provided in Appendix 1.

Pertaining to interprofessional attitudes, student teams reflected on the importance and impact as they related to IPE. One team summarized, "This course was really meant to show us that there is disparity between communication and acceptance between our different professions...In order to serve our patients and our community, we must be open to changes and communication, and prepare to not blame, but instead accept our mistakes and protect ourselves from the same mistake in the future."

Attitudes toward collaboration emerged as a common thread within reflections. Student teams identified the need for a baseline understanding of different perspectives, roles, and responsibilities when working

Table 1. Health Professions Students' Scores on a Validated Instrument ${ }^{\mathrm{a}}$ After Completing an Introductory Interprofessional Education Course $(\mathrm{N}=506)$

\begin{tabular}{|c|c|c|c|c|c|c|c|}
\hline Health Professions Students & $\begin{array}{l}\text { Response } \\
\text { Rate, \% }\end{array}$ & $\begin{array}{c}\text { Retrospective } \\
\text { Pretest, }{ }^{\text {b }} \text { Mean (SD) }\end{array}$ & $\begin{array}{c}\text { Posttest, } \\
\text { Mean (SD) }\end{array}$ & $\begin{array}{l}\text { Absolute Change } \\
\text { Mean, (SD) }\end{array}$ & $t$ & $p$ Value & Cohen's d \\
\hline Dental Hygiene $(n=15)$ & 87 & $36.9(6.88)$ & $40.5(5.25)$ & $3.6(7.38)$ & -1.77 & .103 & 0.59 \\
\hline Health Administration $(n=17)$ & 59 & $37.7(10.75)$ & $39.3(10.89)$ & $1.6(2.63)$ & -1.92 & .087 & 0.15 \\
\hline Nursing $(n=155)$ & 48 & $37.3(8.01)$ & $41.2(8.77)$ & $3.8(6.28)$ & -5.28 & $<.001$ & 0.45 \\
\hline Occupational Therapy $(n=42)$ & 45 & $40.1(5.35)$ & $41.7(5.58)$ & $1.6(2.89)$ & -2.46 & .024 & 0.30 \\
\hline Overall $(n=506)$ & 48 & $36.6(8.09)$ & $40.5(8.57)$ & $3.9(6.07)$ & -10.1 & $<.001$ & 0.47 \\
\hline
\end{tabular}

${ }^{a}$ Student Perceptions of Interprofessional Clinical Education-Revised 2 (SPICE-R2)

${ }^{\mathrm{b}}$ Overall SPICE-R2 score ranged from 0-50 


\section{American Journal of Pharmaceutical Education 2020; 84 (5) Article 7693.}

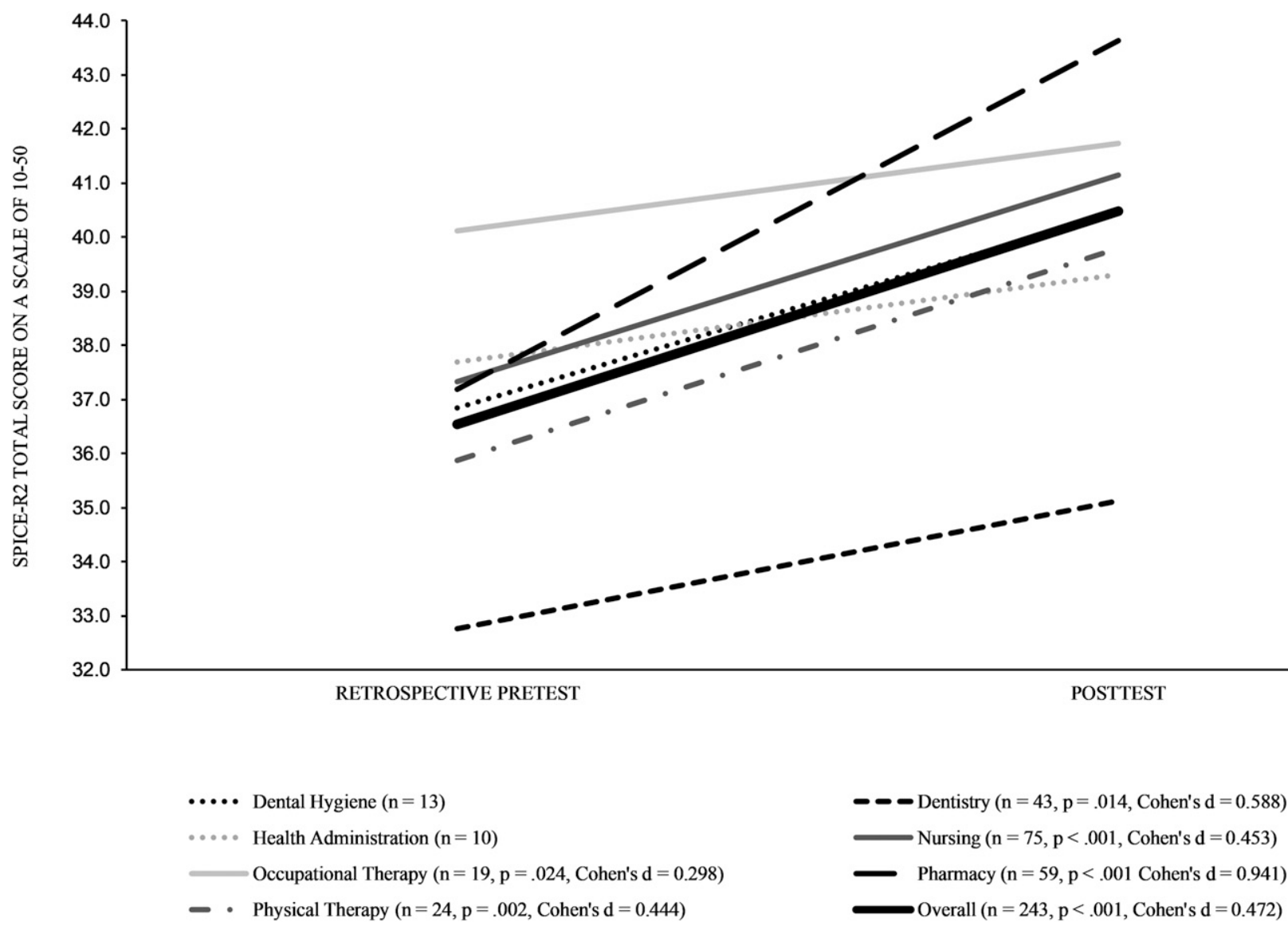

Figure 1. Changes in Overall SPICE-R2 Scores by Student Program and Overall

together in order to provide the best care for patients. The teams highlighted in-class cases and "safety stores" as opportunities to gain appreciation for each other's perspectives and roles, while deepening their understanding of the importance of collaborative communication. In addition to prioritizing collaboration, students were able to understand how the interdependence of their roles extended beyond the classroom and recognize that collaboration improves their ability to provide proper care through appropriate referrals.

Several teams also highlighted the role of bias as a subtheme within the development of their interprofessional attitudes. Course activities allowed teams to identify, acknowledge and overcome common professional stereotypes. Students noted that both courses allowed them to express and identify the impact of their biases and to become more aware of how that bias affects healthcare as a whole. A team commented, "Our stereotypes of the other professions were changed, and we saw how important it could be in the future to rely on other healthcare professionals instead of just our own understanding."

A common subtheme was the idea of a culture of safety, emphasizing the need for a blame-free environment with a shared responsibility toward continual

Table 2. Summary of Themes and Subthemes Identified From Reflections by Teams of Health Professions Students After Completing a Course on Interprofessional Education

\begin{tabular}{|c|c|c|c|c|}
\hline $\begin{array}{l}\text { Interprofessional } \\
\text { Attitudes }\end{array}$ & Communication & $\begin{array}{c}\text { Collaborative } \\
\text { Behavior }\end{array}$ & Professional Identity & Systems of Care \\
\hline Bias & Collaboration & Culture of Safety & Culture of Safety & Culture of Safety \\
\hline Collaboration & Escalating Concerns & Decision Making & Hierarchy & Hierarchy \\
\hline Culture of Safety & Feedback & $\begin{array}{l}\text { Interprofessional } \\
\text { collaboration }\end{array}$ & Overlap of responsibilities & $\begin{array}{l}\text { Impact and design of } \\
\text { technology }\end{array}$ \\
\hline Patient-Centered Care & Managing Conflict & Real-Life Application & Patient-Centered Care & $\begin{array}{l}\text { Models for Improvement } \\
\text { Patient-Centered Care }\end{array}$ \\
\hline
\end{tabular}




\section{American Journal of Pharmaceutical Education 2020; 84 (5) Article 7693.}

evaluation and quality improvement. Many teams highlighted the link between safety and respect in their future practice, identifying the impact that both concepts have on the culture of psychological safety in an organization.

Finally, students also expressed attitudes toward the importance of patient-centered care and an awareness toward a shared end goal for all of their peers. Students were not daunted by the level of effort that interprofessional collaborative care necessitates, identifying that ultimately the patient is the biggest beneficiary of the work. One team reflected, "We are a collaborative team of healthcare professionals dedicated to promoting the overall health and well-being of patients. As with any team, it is essential that each member of the team respect and acknowledge the contributions of their teammates and work together to accomplish the common goal, in this case, being a patient advocate and providing the best possible care." This acknowledgement and understanding of new perspectives and roles that are provided from multiple professions was echoed by several team reflections from both courses.

The second core theme that emerged from student reflections was communication. Teams identified the need for listening and strong communication in providing clinical decisions. Course activities provided students with chances to identify the ideal communication strategies for interprofessional situations. This mindset, and the emphasis on the importance of communication and feedback in providing patient care was echoed in several reflections. From this, several subthemes arose, including giving appropriate feedback, managing conflict, escalating concerns, and enhancing collaboration.

A frequent subtheme of communication was providing appropriate feedback. Activities within the courses provided students the opportunity to witness and critique professional interaction in a simulated setting. Teams emphasized the impact of behavior-based over personbased feedback, identifying the benefits of being open to constructive feedback to further promote collaboration. The interprofessional environment gave students the skills to feel more comfortable providing feedback to each other, across disciplines.

The next two subthemes of communication, managing conflict and escalating concerns, were sometimes discussed separately and sometimes intertwined. Students expected that conflicting viewpoints would arise within the patient care process and that would require conflict management skills. One team commented, "We learned how to effectively communicate and collaborate as [sic] team, respecting each other's questions, concerns, and input. This course helped teach us to navigate these often difficult conversations with our colleagues. That said, in the case where members of the healthcare team are not receptive to these conversations, we discussed in this class various strategies to escalate the issue if it is of significant concern." Regarding escalating concerns, reflection comments indicated an undercurrent of patient safety that students identified as guiding patient care. Teams commented on the importance of a shared understanding between all team members, one that centered around the patient. Reflections indicated an understanding of the importance of speaking up and clarifying concerns, recognizing that patient care could be compromised if team members are not actively communicating with one another.

Finally, team reflections also indicated the importance of promoting collaboration through their communication and feedback. Having a grounded understanding of roles, accepting feedback constructively and working to eliminate stereotypes form the basis for collaboration. One team highlighted the value of quality communication as it related to interprofessional work: "Understanding how each profession works, working cohesively, and communicating in the best way possible can make a huge impact in quality care and safety of our patients."

The third core theme observed across the two courses was collaborative behaviors. This theme was exemplified by the students' reflections on skills or actions they believe should be taken by healthcare professionals and the challenge of developing consensus within a team during the decision-making process.

One subtheme of collaborative behaviors identified the effort needed for interprofessional collaboration. Students reflected on the reality that collaboration extends beyond just working on a single task, but instead involves trust, responsibility and accountability from all involved. Reflections commented on the necessity for exploring alternative perspectives to gain further insight into problems and to avoid errors. Teams were able to understand the context and work necessary for true collaboration, as is evident in this team's reflection: "We believed that these were obvious components to interprofessional practice; it was all common sense. However, when faced with a real-world scenario, such as that of the asthmatic teen, we realized how difficult collaboration towards a goal can be. We had to utilize every skill set available to our group, and define a task that optimized the care the patient received. We put much more energy into the task than just 'common sense,' much more than we would have at the beginning of the course. This course proved to us that interprofessional collaboration is a complex, multi-faceted issue." The sentiments that collaboration takes work was commonly encountered in reflections written for both interprofessional courses. 


\section{American Journal of Pharmaceutical Education 2020; 84 (5) Article 7693.}

A second subtheme focused on decision-making as a collaborative behavior. A class assignment allowed students to use different types of decision-making techniques on a familiar decision. Students commented on the usefulness of understanding each type and when it would be useful in practice, as well as the importance of managing disagreements through collaborative listening. Many reflections described processes of interprofessional decision-making that seemed to overlap with the theme of communication.

Supporting a culture of safety was identified as a third subtheme of collaborative behaviors. Simulation activities utilizing the concepts of escalation of concerns, hierarchies, and system errors provided students with the opportunity to practice and become comfortable with these concepts. Team reflections mentioned the impact of these simulations on their awareness of an overall culture of safety and a desire to collaborate with all members of their future healthcare teams to make the practice environment a safe one. One student reflected, "I see this discussion impacting my future career in that I will be much more aware of the culture of safety which exists and how it is being handled in my practice. Most importantly I will be vigilant about making sure those around me feel comfortable addressing their concerns regardless of their position within the system."

Finally, course evaluations revealed that students perceived the best way to learn interprofessional collaborative behaviors is to apply concepts to real life settings. One student wrote, "I would really have loved to be put to work with other professions. I think the most benefit from interprofessional care should be learned from example not from lectures. I think it would be beneficial to actually see interprofessional care in action, possibly interactions with the fake patients and scenarios the medical school provides or SimMan in the school of pharmacy."

The fourth core theme that emerged from team reflections was the concept of a professional identity. As students learned about roles and responsibilities of various professions through both formal course activities and informal conversations with each other, students recognized the shared goal of patient-centered care, the positives and negatives of overlapping responsibilities, and the impact of professional identity on a culture of safety.

Students acknowledged that overlapping responsibilities can be beneficial toward patient care and ensuring the best health outcomes. Teams commented on the importance of care overlap, such as multidisciplinary rounds, to avoid any gaps in real time care provision. Yet, students also recognized that overlapping responsibilities among professional groups can contribute to conflict as can professional biases and hierarchy in the workplace.
They suggested that knowing one's own professional scope of practice and that of other professions has the potential to break down these barriers toward collaboration. One team reported, "There are stereotypes associated with each profession and it is essential that we acknowledge the existence of these stereotypes, overcome them through education and collaboration, and engage in interprofessional communication. Each healthcare specialty has a specific role and each contributes to the overall health of the patient. We should work to eliminate stereotypes as they are unnecessary and create barriers between healthcare providers and diminish the quality of care to each patient. These stereotypes affect how we view other professions as well as our own, so this can even affect how we operate as individual providers."

A subtheme of developing a culture of safety also emerged from the core theme of professional identity. Students acknowledged the importance of an organizational culture and continuous examination and improvement of its processes. Both transparency and accountability were mentioned by teams as pillars of a culture of safety, extending from individual practitioners all the way to managers and leaders Furthermore, one team wrote, "No matter what role we play within the healthcare system or practice model we participate in, it will be important for us to understand how to identify problems in our practices and to devise ways to perform quality improvement."

The final core theme from student assignments and course evaluations in both courses was a recognition of the role that the healthcare system plays in shaping interprofessional practice. Subthemes within this concept that were identified included patient-centered care, hierarchy, culture of safety, models for improvement of the healthcare system, and the impact of technology and design in the healthcare system. One team discussed how they can improve the healthcare system when they become a part of it: "In a very broad sense, we, as healthcare professionals, will need to create an environment that produces a safe, team-based and positive working environment while also minimizing the potential for harm to come to patients, and also maintaining an efficient and practical healthcare system."

The subtheme of patient-centered care within a complex system was also discussed. Teams recognized that healthcare employees interact for a shared patient goal, regardless of awareness, and that ultimately, support is needed in order to ensure the best outcomes. However, teams also highlighted how the hierarchy of healthcare can interfere with patient-centered care, and the barriers it can cause when others do not feel comfortable sharing their thoughts or concerns due to perceived repercussions or power imbalances. 


\section{American Journal of Pharmaceutical Education 2020; 84 (5) Article 7693.}

The subtheme of a culture of safety within health systems again surfaced as important. As part of the culture of safety, the course discussed models for improvement of the healthcare system. One team reported, "One way we can prevent mistake[s] is by understanding the cause and effect relationship. This should be a key tool in every healthcare professional's mind. We can follow the chain of events and see how different things that lead to one another in medicine to find the cause for a lot of the problems, solve them, and prevent future mistakes in medicine." These models for improvement were described by several groups of learners as important course concepts.

The final subtheme involved the impact of technology and design in the healthcare system. Students highlighted the benefits of computerized and robotic technology as avenues for minimizing medical errors and improving healthcare delivery. Yet, other teams recognized that technology can be a barrier, due to the limited reach of electronic medical records and overreliance on the technology to prevent errors. Many teams highlighted the importance of design and technology to improve safety. One team reported, "Design in hospitals/offices have a significant impact upon patient and staff safety. Even before construction, patient safety is front and center. Luckily, new technology is available in building, designing, and carrying out daily tasks...Tools that are used often in patient rooms and in operating rooms should be designed for people of all ages and sizes."

\section{DISCUSSION}

The purpose of this study was to explore the attitudes of students who complete foundational IPE courses to help us understand how this learning may shape future practice and the composition and timing of IPE. Consistent with other studies, quantitative data demonstrated that student attitudes about interprofessional learning are generally more positive following a foundational IPE course, with some variation correlating with professional differences. ${ }^{18,20}$ However, it is the qualitative findings that provide the most novel and important insights. Based on the themes and subthemes from this analysis, we developed a framework (Figure 2) which has a number of implications for foundational IPE experiences.

These themes are worth comparing and contrasting with current competency frameworks. ${ }^{24,30}$ For example, the communication theme seems to align with the interprofessional communication domain of the Interprofessional Education Collaborative competencies, and the attitudes toward interprofessional practice may align with the values and ethics domain. ${ }^{24}$ The alignment with other competency domains is less straightforward. The roles and responsibilities domain may be subsumed by the professional identity theme, which suggests that learners may think about professional roles and responsibilities in the context of their own and other practitioners' professional identity. Similarly, the teams and teamwork domain may be spread across the themes for collaborative behaviors and systems of care. Those blurry edges between constructs is consistent with challenges outlined in the most recent Interprofessional Education Collaborative report, research examining the domain structure of the competencies, and differing perspectives on how competencies should be classified. ${ }^{24,30-33}$ Notably, the systems of care theme seems least represented in the current conceptualization of interprofessional

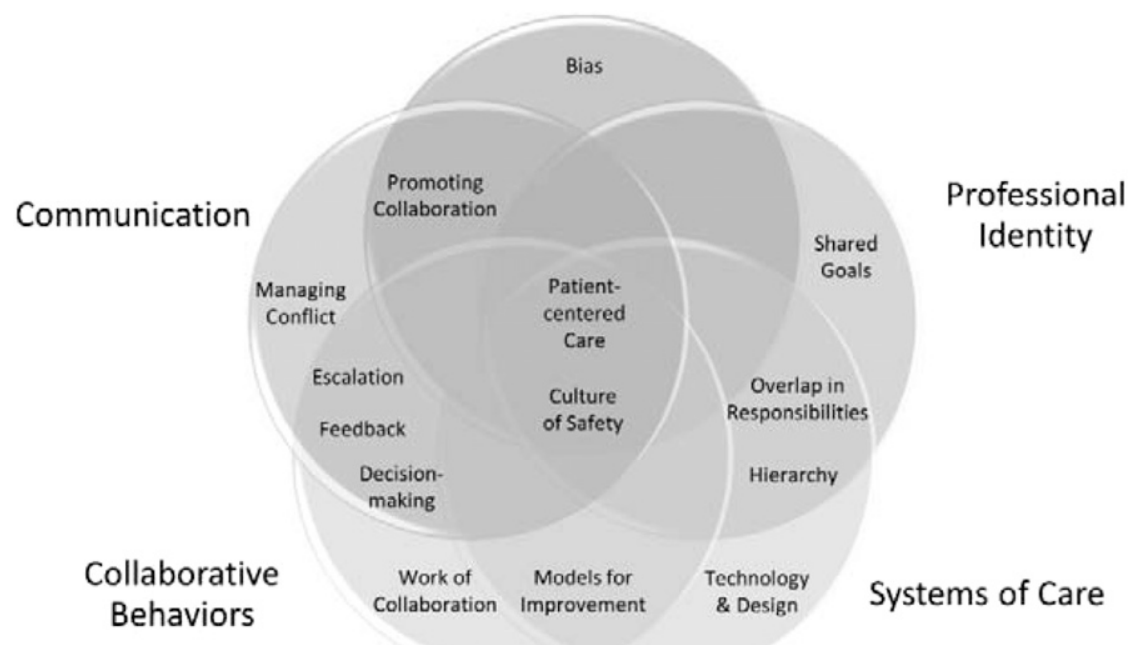

Figure 2. Attitudes to Interprofessional Practice 


\section{American Journal of Pharmaceutical Education 2020; 84 (5) Article 7693.}

competency. Being intentional about the inclusion of systems and how they influence interprofessional practice may be an important addition to competency frameworks and help learners integrate IPE into future practice.

A similar observation can be made based on how students describe the goal of interprofessional practice. While patient-centered care has been rightly defined as the ultimate goal of IPE, students more frequently mentioned the concept of a culture of safety. ${ }^{1}$ This may be the impact of the curriculum on students; however, another possibility is that students, having been acculturated in an educational environment of individual performance, may think about care through the lens of their own individual performance. With such a perspective, the worst occurrence may be making a medical error. If true, IPE has the potential to engage students at their current stage of development by examining interprofessional practice through the lens of medical errors and individual performance rather than patient-centered care. These student attitudes should be further explored.

Students' discussions of the concept of psychological safety, defined as the willingness to take a risk despite potential social consequences, complements our interpretation of their discussions of a culture of safety. ${ }^{32}$ Although students did not always use the specific term of psychological safety, they often alluded to the importance of psychological safety, the role of healthcare professionals in supporting it, and the challenges of acting within a future clinical environment that may or may not have psychological safety. This area seemed to be important for their developing professional identities and how effective interprofessional practice underpins a culture of safety and optimal patient care.

Professional differences may also be important. We identified differences in attitudes across professions in quantitative data. In our sample, pharmacy students exhibited attitudinal scores in the middle of the range of the pretest measure for all students enrolled in the course. Their posttest scores, however, were well above those of students in the other disciplines and represented positive change significantly greater than that of students from dentistry and occupational therapy. While our qualitative analysis did not allow us to make distinctions based on profession, how classroom-based IPE shapes each profession's identity may differ and may be shaped by the type of students attracted to a field, preceding coursework, the culture of a particular program, or societal factors. With early IPE experiences, pharmacy students professional identities may evolve from ones based on solitary work in community-based settings to viewing themselves as part of broad networks of care that span different settings and as integral members of clinical care teams. In contrast, dentistry students may not see as clear a path to a more collaborative professional identity. This issue should be further studied.

Finally, we noted that perceived learning was both quantitatively demonstrated and qualitatively robust. Classroom-based IPE spurred students to think about their future practice and describe how course concepts might integrate into their professional identities. This evidence seems to support the impact of early IPE. Because a controlled trial of a similar approach is unlikely to happen, studies that build on this one with either a longitudinal component or by comparing conceptualization of professional identity to individuals who do not receive IPE may be the best approaches for future work.

There are several limitations of this study. The first and most impactful is the lack of power resulting from small sample sizes among some of the smaller health professions. We conducted a post hoc power analysis with power set at 0.80 and a two-tailed alpha of .05 . The sample sizes from each health profession would have to increase to 30 per group in order to reach statistical significance. Because enrollment and class sizes vary between disciplines, the sample sizes were not able to be adjusted by the study investigators, and the underpowered results may have contributed to the lack of statistical significance found in comparing the survey responses. In addition, the SPICE-R2 was administered using a retrospective pretest-posttest design, which has potential limitations such as recall and self-appraisal bias. The SPICE-R2 has been validated in the literature in studies involving second- and third-year medical and pharmacy students rather than with a full breadth of health care professions students at the start of their learning, which may also limit the application of these results. Thematic analysis was completed using a manual approach versus using computerization because of a lack of funds available to purchase software. Other institutions could repeat or adapt this study using qualitative analysis computer software to not only reduce investigator time but reduce the chance of error or missing themes when sampling from large data sets. Because the reflections were created by teams of students rather than individual students and because the depth of individual participation by students on each team likely varied, the comments submitted and themes identified may not be representative of all students. Similarly, because some of the qualitative data were from course assignments, there could have been some desirability bias. Finally, this analysis examined only one iteration of two courses at a single institution, which may limit generalizability. 


\section{American Journal of Pharmaceutical Education 2020; 84 (5) Article 7693.}

\section{CONCLUSION}

Two classroom-based IPE experiences appeared to affect perceived learning for health professions students and have a significant impact on attitudinal changes for pharmacy students. As we seek to define how IPE affects interprofessional practice and health outcomes, these findings help identify the role of early IPE on professional identity, interprofessional socialization, and, perhaps, intentionality regarding future interprofessional practice. Specifically, how IPE reshapes professional identity for different health professions students is a promising direction for further research on the value of IPE for each health professions degree program. These findings can be used to further develop the theory underlying IPE and new approaches to curriculum, program evaluation, and the development of interprofessional competence among all learners.

\section{REFERENCES}

1. World Health Organization. Framework for Action on Interprofessional Education and Collaborative Practice. https:// www.who.int/hrh/resources/framework_action/en/. Accessed May 14, 2020.

2. Zorek JA, Raehl C. Interprofessional education accreditation standards in the USA: a comparative analysis. J Interprof Care. 2013;27(2):123-30. https://doi.org/10.3109/13561820.2012.718295. Accessed May 14, 2020.

3. Reeves S. Why we need interprofessional education to improve the delivery of safe and effective care. Botucatu. 2016;20(56):185-97. https://doi.org/10.1590/1807-57622014.0092. Accessed May 14, 2020.

4. Institute of Medicine. Measuring the Impact of Interprofessional Education on Collaborative Practice and Patient Outcomes.

Washington, DC: The National Academies Press; 2015.

5. Accreditation Council for Pharmacy Education. Accreditation standards and key elements for the professional program in pharmacy leading to the Doctor of Pharmacy degree. https://www.acpeaccredit.org/pdf/Standards2016FINAL.pdf. Updated January 2015. Accessed May 14, 2020.

6. Meyer BA, Seefeldt TM, Ngorsuraches S, et al. Interprofessional education in pharmacology using high-fidelity simulation. Curr Pharm Teach Learn. 2017;9(6):1055-1062. https://doi.org/10.1016/ j.cpt1.2017.07.015. Accessed May 14, 2020.

7. Kostoff M, Burkhardt C, Winter A, Schrader S. An interprofessional simulation using the SBAR communication tool. Am J Pharm Educ. 2016;80(9):Article 157. https://www.ncbi.nlm.nih.gov/pmc/articles/ PMC5221839/. Accessed May 14, 2020.

8. Shrader S, Kostoff M, Shin T, et al. Using communication technology to enhance interprofessional education simulations. $\mathrm{Am} \mathrm{J}$ Pharm Educ. 2016;80(1):Article 13. https://www.ncbi.nlm.nih.gov/ pmc/articles/PMC4776291/. Accessed May 14, 2020.

9. Fazel MO, Cooley J, Kurdi S, Fazel MA. A co-curricular diabetesspecific elective with interprofessional students and faculty. Curr Pharm Teach Learn. 2019;11(2):172-177. https://doi.org/10.1016/ j.cptl.2018.11.006. Accessed May 14, 2020.

10. Bridgeman MB, Rusay M, Afran J, Yeh DS, Sturgill MG. Impact of an interprofessional medication error workshop on healthcare student perceptions. Curr Pharm Teach Learn. 2018;10(7):975-981. https://doi.org/10.1016/j.cpt1.2018.04.013. Accessed May 14, 2020. 11. Dwivedi R, Cipolle C, Hoefer C. Development and assessment of an interprofessional curriculum for managing diabetes during ramadan. $\mathrm{Am}$ J Pharm Educ. 2018;82(7): Article 6550. https://www.ncbi.nlm.nih.gov/ pmc/articles/PMC6181163/. Accessed May 14, 2020.

12. Theodorou J, Rotz M, Macphail L, et al. Designing and evaluation an interprofessional practice experience involving dental and pharmacy students. Am J Pharm Educ. 2018;82(6): Article 6298. https://www.ncbi.nlm.nih.gov/pmc/articles/PMC6116879/. Accessed May 14, 2020.

13. Woltenberg L, Taylor S. Successes, challenges, and impact of a large-cohort preclinical interprofessional curriculum: a four-year reflection. Curr Pharm Teach Learn. 2018;10(5):662-668. https:// doi.org/10.1016/j.cpt1.2018.01.003. Accessed May 14, 2020. 14. Vouri SM, Grice GR, Roberts A, Berry T, Duncan W. Didactic and experiential interprofessional, inter-institutional courses at a private college of pharmacy. Curr Pharm Teach Learn.

2013;5(6):626-631. https://doi.org/10.1016/j.cptl.2013.07.010.

Accessed May 14, 2020.

15. Paradise E, Whitehead CR. Beyond the lamppost: a proposal for a fourth wave of education for collaboration. Acad Med.

2018;93(10):1457-1463. https://doi.org/10.1097/

ACM.0000000000002233. Accessed May 14, 2020.

16. Lawlis TR, Anson J, Greenfield D. Barriers and enablers that influence sustainable interprofessional education: a literature review. J Interprof Care. 2014;28(4):305-310. https://doi.org/10.3109/ 13561820.2014.895977. Accessed May 14, 2020.

17. Alexandraki I, Hernandez CA, Torre DM, Chretien KC. Interprofessional education in the internal medicine clerkship postLCME standard issuance: results of a national survey. J Gen Intern Med. 2017;32(8):871-876. https://doi.org/10.1007/s11606-017-40043. Accessed May 14, 2020.

18. Blue AV, Chesluk BJ, Conforti LN, Holmboe ES. Assessment and evaluation in interprofessional education: exploring the field. $J$ Allied Health. 2015;44(2):73-82. https://www.ingentaconnect.com/ contentone/asahp/jah/2015/00000044/00000002/art00004. Accessed May 14, 2020.

19. Dow AW. Handbook of Interprofessional Practice: A Guide for Interprofessional Education and Collaborative Care. 1st ed.

Dubuque, IA: Kendall Hunt; 2018.

20. Abu-Rish E, Kim S, Choe L, et al. Current trends in interprofessional education of health sciences students: a literature review. J Interprof Care. 2012;26(6):444-451. https://doi.org/ 10.3109/13561820.2012.715604. Accessed May 14, 2020.

21. Khalili H, Orchard C, Laschinger HK, Farah R. An interprofessional socialization framework for developing an interprofessional identity among health professions students. $J$ Interprof Care. 2013;27(6):448-453. https://doi.org/10.3109/ 13561820.2013.804042. Accessed May 14, 2020.

22. Lockeman KS, Lanning SK, Dow AW, et al. Outcomes of introducing early learners to interprofessional competencies in a classroom setting. Teach Learn Med. 2017;29(4):433-443. https:// doi.org/10.1080/10401334.2017.1296361. Accessed May 14, 2020. 23. Joynes VCT. Defining and understanding the relationship between professional identity and interprofessional responsibility: implications for educating health and social care students. Adv Health Sci Educ Theory Pract. 2018;23(1):133-149. https://doi.org/10.1007/ s10459-017-9778-x. Accessed May 14, 2020.

24. Interprofessional Education Collaborative. Core competencies for interprofessional collaborative practice: 2016 update. https:// 


\section{American Journal of Pharmaceutical Education 2020; 84 (5) Article 7693.}

www.tamhsc.edu/ipe/research/ipec-2016-core-competencies.pdf. Accessed May 14, 2020.

25. Fike DS, Zorek JA, MacLaughlin AA, Samiuddin M, Young RB, MacLaughlin EJ. Development and validation of the student perceptions of physician-pharmacist interprofessional clinical education (SPICE) instrument. Am J Pharm Educ. 2013;77(9):Article 190. https://www.ncbi.nlm.nih.gov/pmc/articles/PMC3831401/. Accessed May 14, 2020.

26. Dominguez DG, Fike DS, MacLaughlin EJ, Zorek JA. A comparison of the validity of two instruments assessing health professional student perceptions of interprofessional education and practice. J Interprof Care. 2015;29(2):144-149. https:// doi.org/10.3109/13561820.2014.947360. Accessed May 14, 2020 .

27. Zorek JA, Fike DS, Eickhoff JC, et al. Refinement and validation of the student perceptions of physician-pharmacist interprofessional clinical education instrument. Am J Pharm Educ. 2016;80(3):47. https://www.ncbi.nlm.nih.gov/pmc/articles/PMC4857642/. Accessed May 14, 2020.
28. Guest G, MacQueen KM, Namey EE. Applied Thematic Analysis. 1st ed. Los Angeles, CA: Sage; 2012.

29. Crabtree BF, Miller WL. Doing Qualitative Research. 2nd ed. Los Angeles, CA: Sage; 1999.

30. Canadian Interprofessional Health Collaborative. A national interprofessional competency framework: 2010February https:// phabc.org/wp-content/uploads/2015/07/CIHC-National-

Interprofessional-Competency-Framework.pdf. Accessed May 14, 2020. 31 . Reeves $\mathrm{S}$. The rise and rise of interprofessional competence. $J$ Interprof Care. 2012;26(4):253-255. https://doi.org/10.3109/ 13561820.2012.695542. Accessed May 14, 2020.

32. Edmondson AC. Learning from failure in health care: frequent opportunities, pervasive barriers. Qual Saf Health Care. 2004;13(2):ii3-9. http://dx.doi.org/10.1136/qshc.2003.009597. Accessed May 14, 2020.

33. Lockeman KS, Dow AW, DiazGranados D, et al. Refinement of the IPEC competency self-assessment survey: results from a multiinstitutional study. J Interprof Care. 2016;30(6):726-731. https:// doi.org/10.1080/13561820.2016.1220928. Accessed May 14, 2020. 


\section{American Journal of Pharmaceutical Education 2020; 84 (5) Article 7693.}

Appendix 1. Additional Exemplars from Student Reflections and Course Evaluations, by Theme

\section{Interprofessional Attitudes}

"It is important to know and learn how to work with individuals from different professions because each profession brings something unique to the table. Knowing and respecting each profession's expertise can help in establishing an interprofessional team that strives for the patient's best health. Working through cases in this class helped to facilitate a better understanding of the roles of different health care workers so that we can all have a greater appreciation for other perspectives."

"The safety stories shared at the beginning of each class have helped us to collaborate together to figure out ways that errors could have been prevented, usually by altering modes and styles of communication as well as by not being afraid to speak up about a potential error when someone feels uneasy."

"Practicing inter-collaboratively not only helps our patients, but helps each healthcare provider learn and be more educated to refer patients to the proper care."

"There are stereotypes associated with each profession and it is essential that we acknowledge the existence of these stereotypes, overcome them through education and collaboration, and engage in interprofessional communication...These stereotypes affect how we view other professions as well as our own, so this can even affect how we operate as individual providers."

"This course helped us better understand how we each play a part in promoting a culture of safety. Although we may play different roles in the healthcare system, we will all be working together and will affect each other's actions."

"Learning about a culture of safety will impact our future careers because it showed us the importance of respect in the workplace among colleagues and superiors and how this influences psychological safety. We learned how important it is for there to be mutual respect between members of a system, regardless of their position, training, or expertise."

\section{Communication}

"We make clinical decisions by listening to each other and communicating and then addressing what each profession can do for the patient and in what order to provide patient centered care."

"Knowing how to communicate with other students and health care professionals encourages us to effectively work together to achieve the best patient outcome possible."

"A key part of interacting with professionals is providing effective feedback that is behavior based and not person based. For example, during medical theater, the pharmacist was being defensive and discrediting the nurse when she gave feedback. Instead, it is useful to be open to professional criticism. Additionally, we need to be mindful of how we communicate feedback to our peers. We need open communication and feedback between different scopes of practice to promote collaboration."

"A culture of safety requires a common understanding between all members of the healthcare team about the need to speak up and the types of situations that threaten patient safety. As future healthcare professionals, we all understand that in our careers we will need to have the courage to speak up when we encounter a situation that has the potential to harm a patient."

"What this class has taught us when considering the complexity of healthcare is that clear, concise communication amongst team members is paramount. If there is any hesitation at all, it is always best to ask and clarify. We have seen multiple times and read countless cases where detrimental errors could have been prevented if team members had simply communicated with one another."

"The base for collaboration is understanding the roles and responsibilities of different professionals, eliminating stereotypes, utilizing effective communication, and providing and receiving constructive feedback."

\section{Collaborative Behaviors}

"Healthcare requires that we work together in order to reduce errors and provide the best care to patients. Healthcare is not going to become simpler but, working as a team, we can attempt to predict where potential errors may occur and prepare for them. We learned that collaboration was more than just cooperating over a task; it involves a deep level of trust, responsibility, and accountability from every member of the interprofessional team. We interact by focusing our abilities and attention to a common goal, achieving optimal holistic care in the process. We learned how alternate perspectives can provide invaluable insight to a problem, often revealing a solution that was previously missed."

"Being able to understand the different types of decision-making along with when to use each type will be extremely beneficial in caring for patients in the future. It will help us avoid any problems with disagreements with other healthcare professionals as we can all take the time to hear everyone's thoughts and ideas and go from there."

"Using systems thinking to evaluate the efficiency of the environment in which healthcare is delivered is important because it notices flaws in the system itself. Once we recognize those flaws, we can take steps to fix them. This moves us away from a system which places blame on individuals who err and ensures that these errors are not repeated. The ultimate goal of a healthcare system is to deliver care in a safe and efficient manner so valuing the design of a system is an important cornerstone of that ideal."

(Continued) 


\section{American Journal of Pharmaceutical Education 2020; 84 (5) Article 7693.}

Appendix 1. (Continued)

Professional Identity

"We realized that although our roles seem to be different, there are many similarities, and ultimately we are all working toward the same goal to provide the best care for the patient. In regards to our similarities, we work in similar settings and a lot of our skills and knowledge overlap. For example, we all are taught to assess in some capacity, and this provides each of us an opportunity to screen and provide preventative therapies or treatments for certain illnesses or injuries. We also educate, whether it be health promotion or prevention/education, on newly diagnosed illnesses or injuries; every member of the collaborative team provides education in some way."

"As proud members of the collaborative health care team, each of the professions has both specific duties and responsibilities, as well as participating in overlaps in care that are critical and necessary for the success and health of the patient. For instance, this can be observed in hospital morning rounds where multiple disciplines are involved in reviewing the patient's care in real time, and devising a treatment plan for moving forward. Included with the medical team are nurses, pharmacists, and social workers. Additionally, occupational therapy and physical therapy are often consulted on mobility and issues related to their ability to successfully complete activities of daily living. As shown in these examples, each member of the health care team is an integral part in ensuring positive patient health outcomes."

"Every member of the team needs to feel comfortable enough to speak up and help provide safety for the patient. We also agree that transparency and accountability are very important as they hold employees and leaders in a system accountable for their actions."

\section{Role of Healthcare System}

"Healthcare is a complex system consisting of teams and health professionals of all different backgrounds and statuses."

"When there is a mistake, our future practices will be transparent and strive to improve our system through the improvement model. We understand that this improvement may require changing designs of the system, because human error is only partially responsible for the majority of mistakes. These design flaws underline the complexity of healthcare. Even when we make the proper designs into the system, errors will find a way through, like holes liming [sic] up with slices of swiss cheese."

"Healthcare employees interact and collaborate through a mutual patient even if they do not realize this,"

"There are current barriers to teamwork in the hospital based on the hierarchy system in which many people are afraid of repercussions of going above one of their superiors."

"We also understand both the importance of the hierarchy and the way it can also inhibit patient care within the health care setting."

"[T]he culture of safety is [an] essential component of reducing errors and is needed to improve overall healthcare quality. This was seen in our safety stories that we discussed. It seemed that most of these errors could have been prevented if a culture of safety was in place where providers felt more comfortable to speak up."

"Computer systems can be used as an aid to improve the delivery of healthcare in an effort to minimize and prevent medical errors. For example, most of our future practice includes using computers and robots to do things for us, but we have to be cautious to avoid and prevent medical errors from occurring."

"... the fact that different computer systems were used between the professions was surprising. It provides more insight to when we all become healthcare professionals to be more mindful of things such as medication errors that may arise due to computer systems."

"The impact of design is an important consideration for hospitals and pharmaceutical companies alike. Products are packaged by manufacturers with efficiency and the financial bottom line in mind, however, the similarity or ambiguousness of pharmaceutical packaging is a breeding ground for mistakes by those administering the drugs." 